\title{
Celastrol induces apoptosis of human osteosarcoma cells via the mitochondrial apoptotic pathway
}

\author{
XIAOLONG YU, XIN ZHOU, CHANGLIN FU, QIANG WANG, TAO NIE, FAN ZOU, \\ RUNSHENG GUO, HUCHENG LIU, BIN ZHANG ${ }^{*}$ and MIN DAI*
}

Department of Orthopedics, The First Affiliated Hospital of Nanchang University, Nanchang, Jiangxi 330006, P.R. China

Received April 29, 2015; Accepted June 15, 2015

DOI: $10.3892 /$ or.2015.4124

\begin{abstract}
Celastrol is an active compound extracted from the root bark of Triptergium wilfordii Hook F., also known as 'Thunder of God Vine'. It is a well-known Chinese medicinal herb that was found to inhibit tumor cell growth and promote apoptosis in several tumor cell lines. However, research into its effects on osteosarcoma cell apoptosis is still extremely limited. The present study was undertaken to determine the effect of celastrol on viability and apoptosis of osteosarcoma cells and furthermore, to illuminate the molecular mechanism of celastrol-induced osteosarcoma cell apoptosis. The 3-(4,5-dimethylthiazol-2-yl)-2,5-diphenyltetrazolium bromide (MTT) colorimetric assay was used to evaluate the viability of the cells following treatment with celastrol. The effect of celastrol on the apoptotic rate of the cells was evaluated by flow cytometry using Annexin V-PE/7-AAD staining assay. Fluorescence microscopy was used to detect the morphological changes in the human osteosarcoma U-2OS cell lines. The expression of Bcl-2 family proteins, caspase- 3 , caspase- 8 , caspase-9, cytochrome $\mathrm{c}$ and PARP was measured by western blotting. We found that celastrol significantly inhibited the growth of osteosarcoma cells in a dose-dependent manner, particularly U-2OS cells. Furthermore, we observed that celastrol upregulated the expression of the pro-apoptotic proteins Bax and cytochrome $\mathrm{c}$ and altered the ratio of Bax/Bcl-2, and triggered the mitochondrial apoptotic pathway, resulting in caspase- 3 and -9 activation and PARP cleavage. To conclude, the results indicate that celastrol inhibits the proliferation of human osteosarcoma cancer cells by inducing apoptosis via the mitochondrial-dependent pathway.
\end{abstract}

Correspondence to: Dr Bin Zhang or Min Dai, Department of Orthopedics, The First Affiliated Hospital of Nanchang University, 17 Yong Wai Zheng Street, Nanchang, Jiangxi 330006, P.R. China

E-mail: 1207672246@qq.com

E-mail: 1135547344@qq.com

*Contributed equally

Key words: celastrol, human osteosarcoma, anticancer activity, apoptosis, mitochondrial pathway

\section{Introduction}

Osteosarcoma is the most common primary malignant tumor of the bone predominantly occurring in childhood and adolescence. Among individuals younger than 20 years of age, the osteosarcoma incidence rate is 8.7 per million and the risk is higher in males (1). It usually occurs in a growing long bone such as the humerus, femur or tibia. The current treatment for osteosarcoma is neoadjuvant chemotherapy, surgical resection and chemotherapy again following surgery (2). Despite the advances in treatment options, recurrence and chemoresistance have been the main challenges confronting physicians (3-5). In patients with localized osteosarcoma, the 5-year survival rates are $\sim 65-75 \%(6,7)$. However, the prognosis for patients with recurrence and metastases is quite poor, with 5-year survival rates ranging from 15 to $30 \%$ (8). In addition, most neoadjuvant chemotherapy drugs carry the risk of uncertain effectiveness and severe side effects, and multidrug resistant cases are common, particularly with cisplatin and doxorubicin (9). Over the past 35 years, there has been no significant improvement in chemotherapy for osteosarcoma (10). Therefore, a better understanding of the molecular mechanisms involved in osteosarcoma progression should help to explore novel therapeutic targets or develop new modalities of osteosarcoma therapy. Recently, research into osteosarcoma treatment has been focused on novel target therapies including induction of apoptosis and reduction of cell growth in osteogenic sarcoma (11-13).

Traditional Chinese medicine is an important type of complementary and alternative medicine, because it has a standardized system of diagnostics and therapies, and is practised worldwide (14). Some plant extracts used in complementary medicine, exert potent anticancer activity with low toxicity. Celastrol was found to inhibit growth and accelerate apoptosis in many human cancer cell lines such as hepatoma, breast, myeloma, pancreas and gastric cancer cell lines (15-19).

Apoptosis is a strictly controlled mechanism of cell suicide that is triggered by certain internal or external signals. It results in cell rounding and shrinkage, chromatin condensation, DNA fragmentation and shedding of smaller fragments from cells. In the intrinsic pathway, mitochondria play a central role in the occurrence of apoptosis induced by many chemotherapeutic agents (20-22). Mitochondrial membrane permeabilization, along with the collapse of electrochemical gradient across the mitochondrial membrane leads to the release of catabolic hydro- 
lases and activators of some enzymes from the mitochondria, resulting in cell apoptosis $(23,24)$. Bcl-2 family proteins serve as crucial regulators of this pathway through their influence on mitochondrial outer membrane permeabilization (MOMP) following homo- or hetero-association $(25,26)$. Among Bcl-2 family proteins, pro-apoptotic proteins such as Bax, Bad and Bid, increase MOMP during apoptosis and release apoptogenic proteins into the cytosol, such as cytochrome $c$, which can bind to Apaf-1 and further activate caspase-9. Furthermore, activated caspase-9 activates downstream caspase-3 and/or -7, which in turn results in the cleavage or degradation of several key cellular substrates, including PARP, thus leading to apoptosis (27-30).

However, anti-apoptotic proteins such as Bcl-2 and Bcl-XL, can bind to activated Bax to decrease membrane permeability (31). The regulation of activated anti- and pro-apoptotic Bcl-2 family members is essential for determining the fate of cells, and disturbance of the normal apoptotic program due to alteration of the ratio by aberrant expression of these proteins may cause various apoptosis-related diseases $(32,33)$. In addition, Bcl-2 and Bcl-XL overexpression, widely observed in various types of cancers, inhibits apoptosis and confers resistance to anticancer drugs $(34,35)$. Therefore, induction of apoptosis through the mitochondrial-dependent pathway has been one of the targets of anticancer chemotherapy.

\section{Materials and methods}

Materials and reagents. Dulbecco's modified Eagle's medium (DMEM), fetal bovine serum (FBS), phosphate-buffered saline (PBS) and dimethyl sulphoxide (DMSO) were provided by Transgen (Beijing, China). A Hoechst 33258 staining kit was provided by Keygen Biotech (Nanjing, China). MTT [3-(4,5-dimethylthiazol-2-yl)-2,5-diphenyltetrazolium bromide] was obtained from Solarbio (Beijing, China). Antibodies against Bcl-2, Bax, caspase- 3 , caspase- 8 , caspase- 9 and $\beta$-actin were purchased from Abcam (Cambridge, UK), and antibodies against PARP and cytochrome $c$ were purchased from Cell Signaling Technology (Beverly, MA, USA). Horseradish peroxidase (HRP)-conjugated secondary antibodies were purchased from Cell Signaling Technology and Transgen. An Annexin V-PE/7-AAD apoptosis detection kit was provided by Becton-Dickinson (San Jose, CA, USA). Celastrol was obtained from Nanjing Zelang Medical Technology Co., Ltd. (Nanjing, China). Stock solutions of celastrol were prepared by dissolving the celastrol powder in DMSO to a concentration of $1 \mathrm{M}$, and stored at $-20^{\circ} \mathrm{C}$. The working concentrations of celastrol were made by diluting the stock solution with the culture medium. The final concentration of DMSO in the medium was $<0.5 \%$.

Cell culture. Human osteosarcoma cell lines, MG-63 (wild-type), U-2OS (wild-type) and HOS (wild-type), were obtained from the American Type Culture Collection (ATCC; Manassas, VA, USA). Cells were cultured in DMEM supplemented with $10 \%$ (v/v) FBS, $100 \mathrm{U} / \mathrm{ml}$ penicillin and $100 \mu \mathrm{g} / \mathrm{ml}$ streptomycin. They were all placed in a humidified atmosphere containing $5 \% \mathrm{CO}_{2}$ at $37^{\circ} \mathrm{C}$. The cells used were subjected to $<20$ cell passages and were in the logarithmic growth phase.

Cell viability by MTT assay. The cells were cultured in 96-well plates at a concentration of $1 \times 10^{4}$ cells/well. Cell viability was determined using an MTT colorimetric assay. The cells were treated with celastrol at various final concentrations $(0.5,1,2$, 4 and $6 \mu \mathrm{M}$, respectively), for 24,36 and $48 \mathrm{~h}$, and the control cells were treated with $0.5 \%$ DMSO. After the indicated cultivation time, $50 \mu \mathrm{l}$ of MTT $(5 \mathrm{mg} / \mathrm{ml}$ in PBS) was added and the plates were incubated at $37^{\circ} \mathrm{C}$ for an additional $4 \mathrm{~h}$. Finally, the formazan precipitate was dissolved in $100 \mu \mathrm{l}$ DMSO and the cells were shaken for $10 \mathrm{~min}$. Absorbance was measured at $490 \mathrm{~nm}$ using a Universal microplate reader (EL800; BioTek Instruments Inc.). ELISA reader (BioTek, Model EXL800; USA). Cell growth expressed as percent viability was calculated by comparing the absorbance of treated vs. untreated cells.

Hoechst 33258 staining of U-2OS cells. Cells were incubated with $0,1,2.5$ and $4 \mu \mathrm{M}$ of celastrol for $48 \mathrm{~h}$, harvested, fixed with $4 \%$ paraformaldehyde for $30 \mathrm{~min}$ at $25^{\circ} \mathrm{C}$, washed 3 times with ice-cold PBS and stained with $10 \mathrm{mg} / \mathrm{l}$ Hoechst 33258 (Sigma) for $10 \mathrm{~min}$ in the dark at room temperature. Finally, the stained nuclei were observed under a fluorescence microscope (Olympus, $\mathrm{x} 100$ ) with excitation at $350 \mathrm{~nm}$ and emission at $460 \mathrm{~nm}$.

Analysis of cell apoptosis by Annexin V-PE/7-AAD staining assay. To assess the development of apoptosis induced by celastrol, U-2OS cells were stained with Annexin V-PE/7-AAD (BD Biosciences, San Jose, CA, USA). U-2OS cells (1x10 $)$ were cultured in 12-well plates. Following overnight incubation, these cells were treated with celastrol at various concentrations for $48 \mathrm{~h}$ and collected by trypsinization, not containing EDTA. After being twice washed with $4^{\circ} \mathrm{C}$ PBS, the cell pellets were suspended again in $400 \mu \mathrm{l}$ ice-cold $1 \mathrm{X}$ binding buffer at a density of nearly $1 \times 10^{6}$ cells $/ \mathrm{ml}$, and then incubated with $10 \mu \mathrm{l}$ Annexin V-PE/7-AAD for $10 \mathrm{~min}$ in the dark at room temperature. Samples were analyzed by a flow cytometer within $1 \mathrm{~h}$ of staining.

Western blot analysis. U-2OS cells were cultured in 6-well plates at a concentration of $2 \times 10^{5}$ cells/well. After treatment with celastrol at various concentrations for $48 \mathrm{~h}$, the cells were collected and lysed in RIPA buffer containing a protease inhibitor cocktail (Sigma Chemical, USA). The homogenates were centrifuged at $12,000 \mathrm{rpm}$ for $10 \mathrm{~min}$ at $4^{\circ} \mathrm{C}$ and the supernatant fraction was collected for immunoblotting. Furthermore, protein concentrations were calculated by a BCA assay using bovine serum albumin as the standard. The same amounts of proteins were loaded and separated by electrophoresis on $12 \%$ SDS-polyacrylamide gels under a reducing condition using $100 \mathrm{~V}$ for $2 \mathrm{~h}$. After electrophoresis, the proteins were transferred to PVDF membranes in a Tris-glycine transfer buffer using a semi-dry blotting system, and incubated with antibodies against Bcl-2, Bax, cytochrome $c$, PARP, caspase-3, caspase- 8 , caspase- 9 and $\beta$-actin $(1: 1,000)$ overnight at $4^{\circ} \mathrm{C}$. After PVDF membranes were washed in TBST 3 times, secondary HRP-conjugated antibodies were added at 1:2,000 dilution for $1 \mathrm{~h}$ at room temperature and the PVDF membranes were washed again in TBST 3 times. Immunoreactive proteins were detected by enhanced chemiluminescence (ECL kit; Transgen) followed by exposure to X-ray film.

Statistical analysis. Data were analyzed using the SPSS package for Windows (version 17.0). Quantitative data are 
A

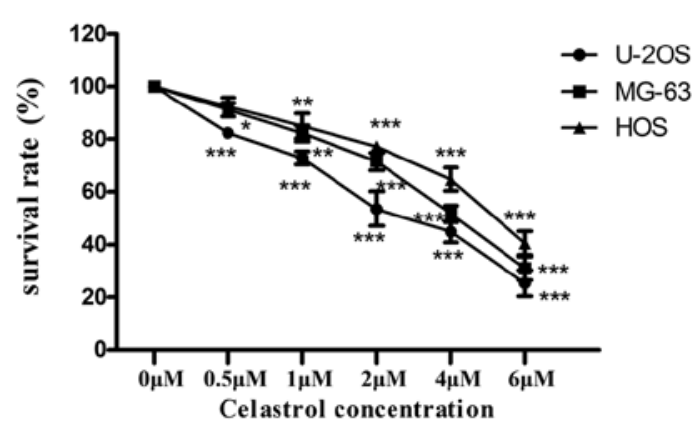

B

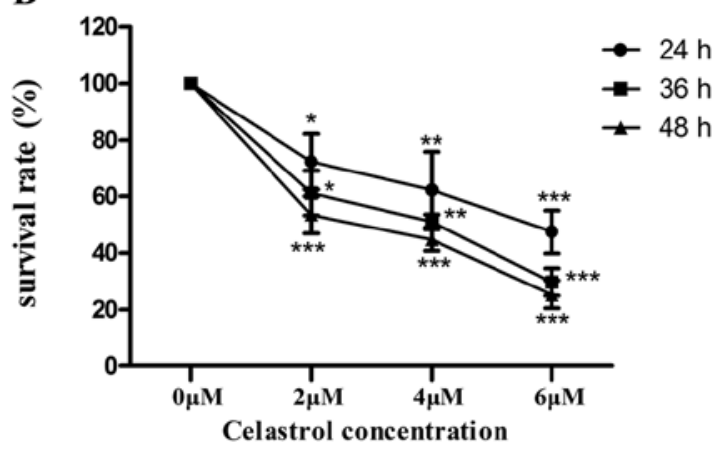

Figure 1. Analysis of cell viability. (A) Effect of celastrol on the viability of MG-63, U-2OS and HOS cells. Cells were treated with celastrol at different concentrations for $48 \mathrm{~h}$. (B) Dose- and time-effect of celastrol on the viability of U-2OS cells. Cells were treated with celastrol at different concentrations for 24,36 and 48 h. Cell viability was then determined and expressed as means \pm SD. Significant differences from control $(0 \mu \mathrm{M})$ are indicated by ${ }^{*} \mathrm{p}<0.05,{ }^{* *} \mathrm{p}<0.01,{ }^{* * *} \mathrm{p}<0.001$.

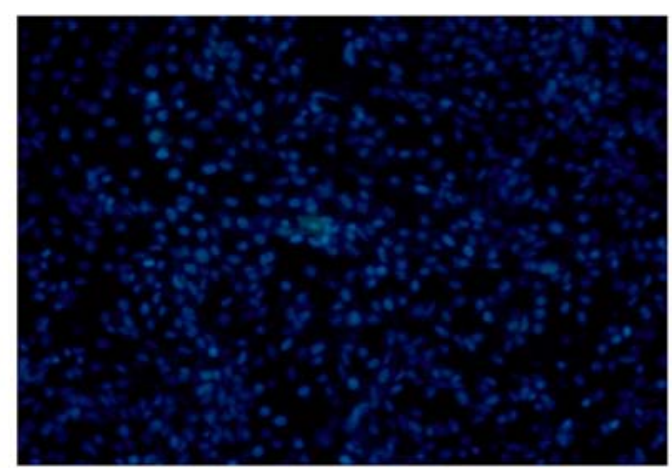

$0 \mu \mathrm{M}$

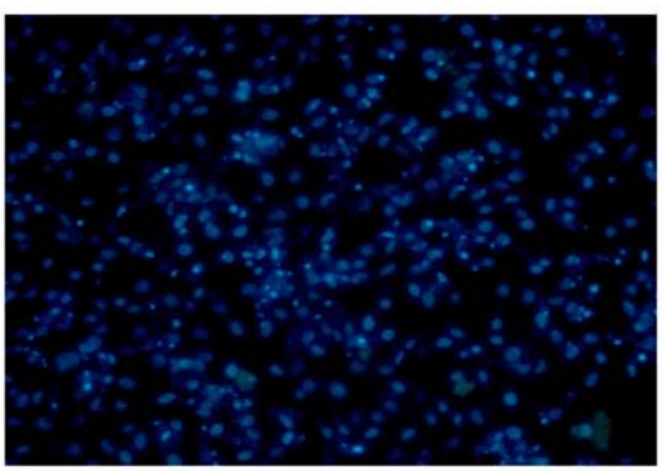

$2.5 \mu \mathrm{M}$

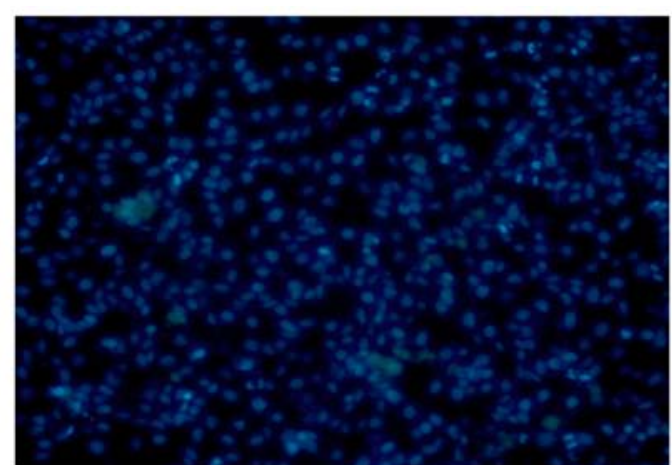

$1 \mu \mathrm{M}$

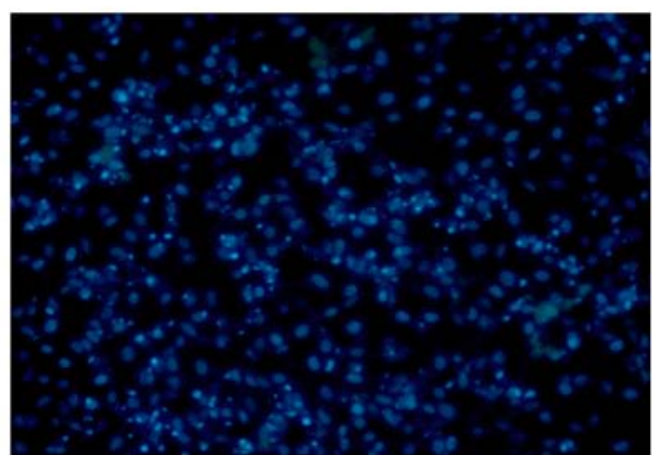

$4 \mu \mathrm{M}$

Figure 2. Hoechst 33258 staining of U-2OS cells. Apoptotic nuclei manifested condensed or fragmented DNA brightly stained by Hoechst 33258 ( 48 h) (magnification, x100).

expressed as the mean \pm standard deviation (SD). Statistical analysis of the data was performed using a Student's t-test and ANOVA. P-values of $<0.05$ were considered to be statistically significant.

\section{Results}

Celastrol reduces the viability of $U-2 O S$ cells. The effect of celastrol on the viability of osteosarcoma cell lines was determined by MTT assay, and we treated three human osteosarcoma cell lines (MG-63, U-2OS and HOS) with celastrol at different concentrations for 24,36 and $48 \mathrm{~h}$, respectively. As shown in Fig. 1A, the inhibitory effects of celastrol on the human osteosarcoma cell lines were dose-dependent, but each cell line exhibited a different sensitivity to celastrol. Obviously, the U-2OS cells were the most sensitive to celastrol. The $\mathrm{IC}_{50}$ value for the U-2OS cells treated with celastrol was $2.5 \mu \mathrm{M}$ at $48 \mathrm{~h}$. As shown in Fig. 1B, the inhibitory effects of celastrol on the human osteosarcoma cell lines was time-dependent. Furthermore, U-2OS cells were treated with celastrol at the concentrations of $0,1,2.5$ and $4 \mu \mathrm{M}$ for $48 \mathrm{~h}$ in the following assays. Our findings demonstrated that celastrol 
A

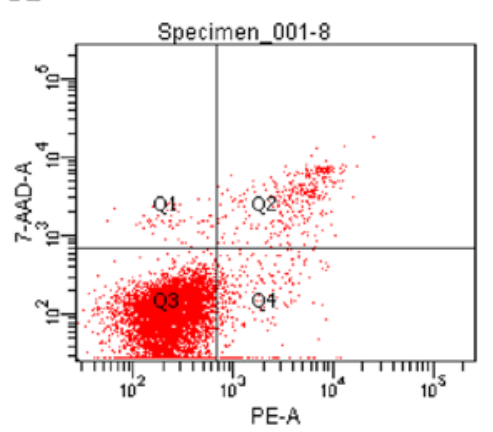

$\mathbf{0} \mu \mathrm{M}$

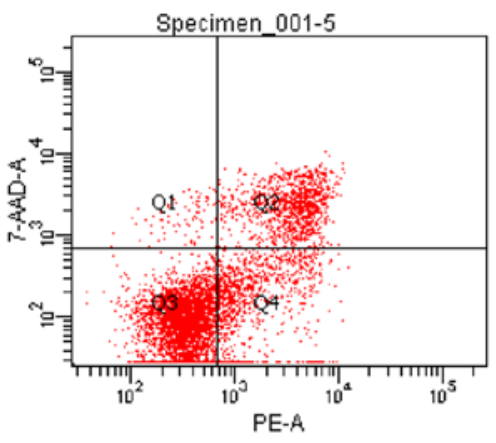

$2.5 \mu \mathrm{M}$

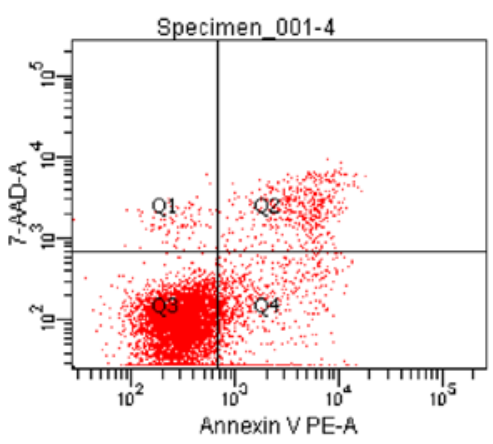

$1 \mu \mathrm{M}$

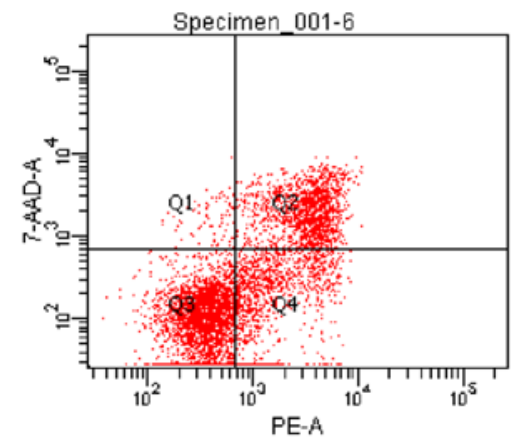

$4 \mu \mathrm{M}$

B

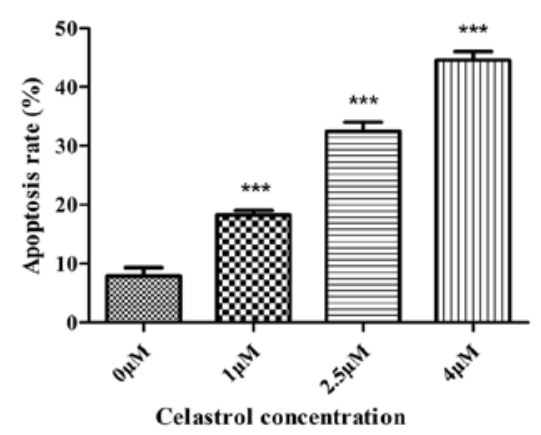

Figure 3. Annexin V-PE/7-AAD staining assay. (A) Representative graphs obtained by flow cytometric analysis after double-staining with Annexin V-PE/7-AAD (B) Compared with the control cells $(0 \mu \mathrm{M}, 7.9 \pm 1.4 \%)$, the percentage of apoptotic U-2OS cells was increased to $18.2 \pm 0.8,32.5 \pm 1.6$ and $44.6 \pm 1.4 \%$ in a dose-dependent manner after treatment for $48 \mathrm{~h}$. Significant differences from control $(0 \mu \mathrm{M})$ are indicated by ${ }^{*} \mathrm{p}<0.05,{ }^{* *} \mathrm{p}<0.01,{ }^{* * *} \mathrm{p}<0.001$.

inhibited cellular proliferation in a time- and dose-dependent manner.

Induction of morphological changes of $U$-2OS cells. Untreated $\mathrm{U}-2 \mathrm{OS}$ cells grew well as observed by phase contrast microscopy. After $48 \mathrm{~h}$ of treatment, celastrol produced broken, necrotic and detached cells in a dose-dependent manner, which was consistent with the growth inhibition. Celastrol-treated U-2OS cells stained with the fluorescent DNA-binding dye Hoechst 33258 revealed condensed and fragmented nuclei, which are typical morphological features of apoptotic cells. In contrast, no morphological signs of apoptosis were observed in the untreated cells. The results indicated that cell death occurred through apoptosis (Fig. 2).

Annexin V-PE/7-AAD staining assay. The rate of cell apoptosis was detected by flow cytometry following double labeling with Annexin V-PE/7-AAD. Representative graphs obtained by flow cytometric analysis of the cells treated with celastrol at different concentrations for $48 \mathrm{~h}$ after double staining with Annexin V-PE and 7-AAD are shown in Fig. 3A. The apoptosis rate in the control cells was $7.9 \pm 1.4 \%$. There was a dose-dependent increase in the apoptosis rate of U-2OS cells treated with celastrol. The apoptosis rates in the U-2OS cells were increased to $18.2 \pm 0.8,32.5 \pm 1.6$ and $44.6 \pm 1.4 \%$ following treatment with celastrol at 1, 2.5 and $4 \mu \mathrm{M}$ for $48 \mathrm{~h}$, respectively (Fig. 3B).

Celastrol decreases the expression of anti-apoptotic Bcl-2 and increases the expression of pro-apoptotic Bax and cytochrome $c$. To determine the molecular mechanism by which celastrol induces the apoptosis of U-2OS cells, the protein expression levels of Bcl-2 family proteins, including anti-apoptotic members such as Bcl-2, and pro-apoptotic members such as Bax and cytochrome $c$, were assessed by performing 

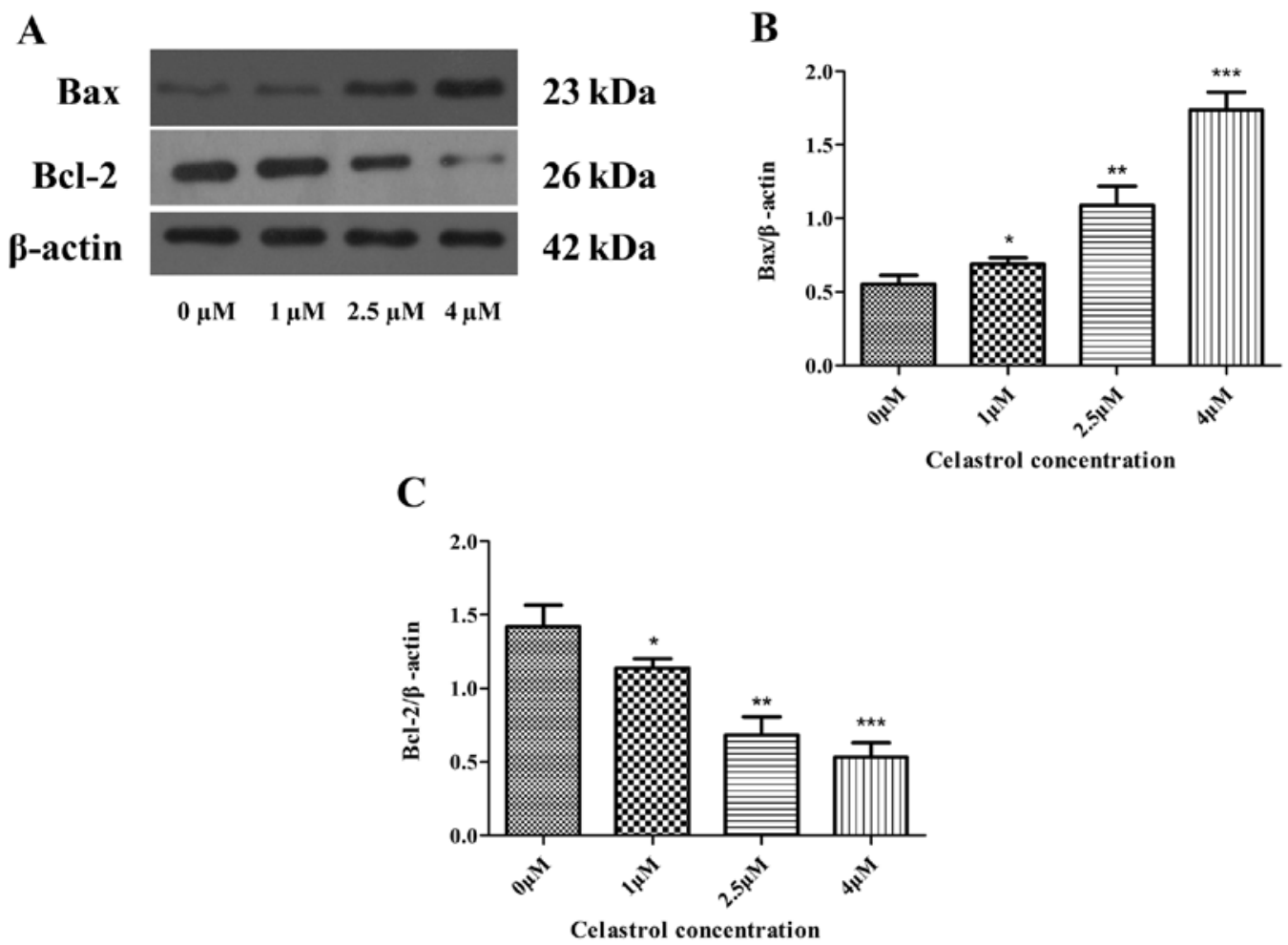

Figure 4. Effect of celastrol on the protein expression levels of Bcl-2 and Bax in U-2OS cells. (A) The protein expression levels of Bax and Bcl-2 were analyzed by western blot analysis. $\beta$-actin was used as the internal control for the western blot analysis. (B and C) Quantification of western blot analysis. The protein expression levels of Bax (B) and Bcl-2 (C) in celastrol-treated and control cells. The data shown are the averages $\pm \mathrm{SD}$ (error bars), ${ }^{*} \mathrm{p}<0.05,{ }^{* *} \mathrm{p}<0.01$, **** $\mathrm{p}<0.001$, significant vs. control cells.

western blot analysis. The results of the western blot analysis revealed that celastrol treatment caused a profoundly marked increase in Bax proteins and the release of cytochrome $c$, and a decrease in $\mathrm{Bcl}-2$ protein, when compared to these levels in the control (Figs. 4A-C and 5A and B). This demonstrates that celastrol activates the mitochondrial apoptotic pathway in $\mathrm{U}-2 \mathrm{OS}$ cells via regulating the expression of the Bcl-2 family proteins.

Effects of celastrol on the expression levels of caspases. The caspase cascade reaction is one of the most important events in the process of apoptosis through the mitochondrial pathway. Therefore, the protein expression levels of caspase- $3,-8$ and procaspase- 9 were assessed by performing western blot analysis (Figs. 5A and 6A). Caspase-3 cleavage was observed (Fig. 6A and B), and expression levels of procaspase-9 were downregulated, both in a concentration-dependent manner as the concentration of celastrol increased (Fig. 5A and C). However, expression levels of caspase- 8 were not changed in the cells treated with celastrol (Fig. 5A and D). Cleavage of PARP, a key cellular substrate, was observed (Fig. 6C and D). The results indicated that the apoptosis induced by celastrol involved the caspase cascade and was triggered through the mitochondrial pathway.

\section{Discussion}

Apoptosis, a program of cell suicide, is an innate cellular response to eliminate abnormal or redundant cells in mammals and hence is considered an important mechanism in the action of many anticancer drugs (22). There is accumu- lating evidence that a wide variety of herbal medicines and compounds extracted from natural products with antitumor effects can trigger apoptosis in various tumor cells (20-22). Previous studies have demonstrated that celastrol, a triterpene extracted from the root bark of Triptergium wilfordii Hook F., also known as 'Thunder of God Vine, can inhibit tumor promotion (15-19). In the present study, we determined the anticancer effect and associated mechanisms of celastrol on human osteosarcoma cells lines in vitro. MTT results revealed that celastrol effectively suppressed the proliferation of three human osteosarcoma cell lines (MG-63, U-2OS and HOS) in a dose- and time-dependent manner. FACS analysis showed that celastrol effectively induced apoptosis in the osteosarcoma cells. Thus, we next investigated the apoptotic mechanism of celastrol on osteosarcoma cells.

Apoptosis is triggered by two different signals: the mitochondrial pathway and the cell death receptor pathway, regulated via caspase- 9 and -8 , respectively (36). Accumulated evidence has shown that caspases play critical roles in the apoptotic cascade. In the mitochondrial pathway (the intrinsic pathway), downstream of caspase activation is regulated by members of the Bcl-2 family. Apoptosis-associated MOMP is known to require pro-apoptotic Bax-like proteins, in the regulation of pore formation in mitochondria. Anti-apoptotic Bcl-2-like proteins in mitochondrial morphogenesis are functionally distinct from their role in apoptosis. Therefore, the ratio of $\mathrm{Bax}$ to $\mathrm{Bcl}-2$ is vital for determining the release of many apoptogenic proteins from the mitochondrial intermembrane space, such as cytochrome $c$ which can further activate caspase-9. Activated caspase- 9 then activates down- 
$\mathbf{A}$

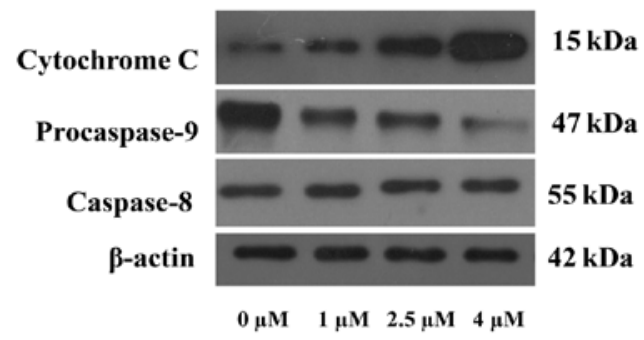

C

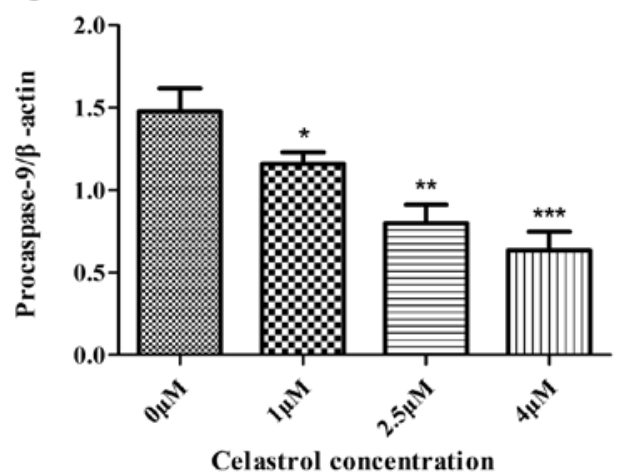

B

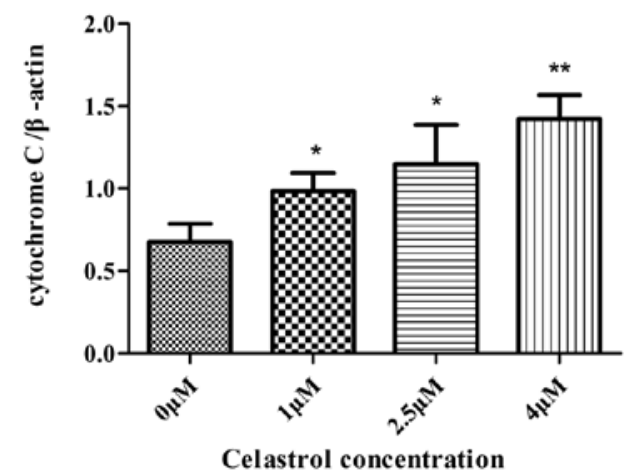

D



Celastrol concentration

Figure 5. Effect of celastrol on the protein expression levels of cytochrome $c$, procaspase-9 and caspase-8 in U-2OS cells, analyzed by western blot analysis (A) The expression levels of cytochrome $c$ were significantly upregulated, while the levels of procaspase- 9 were downregulated, but a change in caspase- 8 expression levels was not observed. $\beta$-actin was used as the internal control for the western blot analysis. (B-D) Quantification of western blot analysis. The protein expression levels of cytochrome $c(B)$, procaspase-9 (C) and caspase-8 (D) in celastrol-treated and control cells. The data shown are the averages \pm SD (error bars), and significant differences from control cells are indicated by ${ }^{*} \mathrm{p}<0.05,{ }^{* *} \mathrm{p}<0.01,{ }^{* * *} \mathrm{p}<0.001$.

A

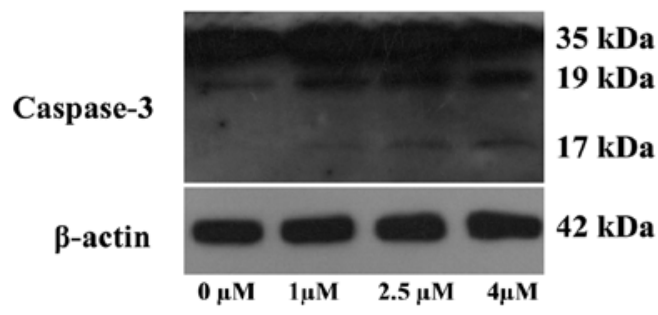

B

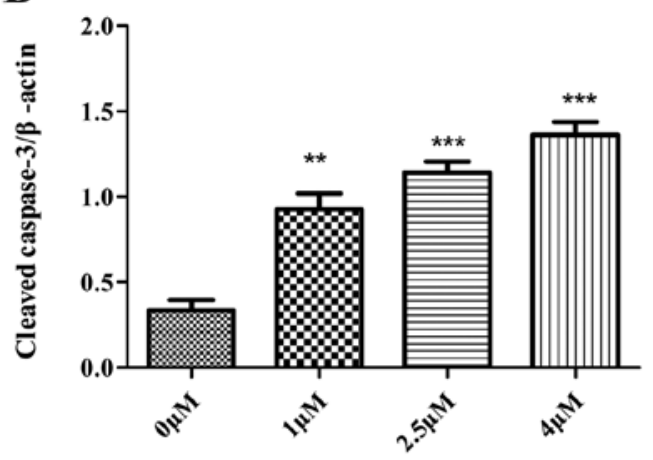

Celastrol concentration
C

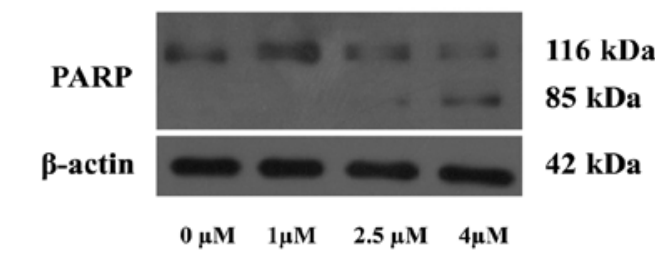

D

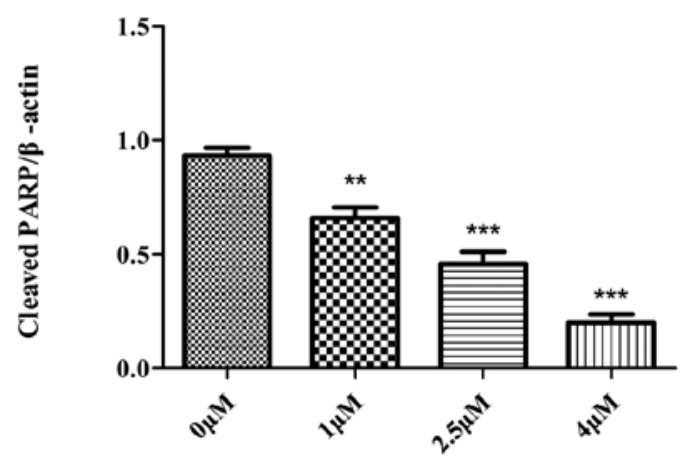

Celastrol concentration

Figure 6. Effect of celastrol on the protein expression levels of caspase-3 and PARP in U-2OS cells. (A and C) Western blot analysis was performed and $\beta$-actin was used as the internal control. Cleavage of caspase-3 and PARP was observed. (B and D) Quantification of western blot analysis. The protein expression levels of cleaved caspase-3 (B) and cleaved PARP (D) in celastrol-treated and control cells. The data shown are the averages \pm SD (error bars), ${ }^{* *} \mathrm{p}<0.01$, ${ }_{* * * *}^{*} \mathrm{p}<0.001$, significant vs. control cells. 
stream caspase-3, which causes the cleavage or degradation of various key cellular substrates, including PARP, thus resulting in apoptosis (27-30,37-39). The cell death receptor pathway (the extrinsic pathway) activates the death receptor on the cell surface (Fas/FasL) and then promotes caspase- 8 activation (40-42). With this in mind, to demonstrate which signaling pathway is involved in apoptosis by celastrol, expression of Bcl-2 family proteins, caspase-3, -8 and -9 and PARP were assessed in U-2OS cells. The present data showed that celastrol-induced apoptosis was accompanied by alteration of the Bax/Bcl-2 ratio and activation of caspase-3 and -9 , but not of caspase-8. Furthermore, cleavage of PARP was also observed. These findings indicated that celastrol-induced apoptosis in $\mathrm{U}-2 \mathrm{OS}$ cells was triggered by an intrinsic pathway.

In conclusion, we demonstrated that celastrol dose-dependently upregulates Bax expression and downregulates Bcl-2 expression in U-2OS cells. This results in the release of cytochrome $c$ into the cytosol, which further activates caspase-9. Furthermore, activated caspase- 9 activates downstream caspase- 3 which in turn, results in the cleavage or degradation of several key cellular substrates, including PARP, and leads to the subsequent apoptosis. These results indicated that celastrol could be a potential novel therapeutic agent for the treatment of osteosarcoma. Further studies are required in order to ascertain whether celastrol can synergize with other chemotherapy drugs. In addition, studies on the in vivo effect of celastrol on $\mathrm{U}-2 \mathrm{OS}$ xenograft tumors in nude mice are in progress.

\section{Acknowledgements}

This study was supported by the Natural Science Foundation of Jiangxi Province (20132BAB205081), the Foundation of the Health Department of Jiangxi Province on Traditional Chinese Medicine (2012A136) and the Engineering Technology Research Center Construction Project of Jiangxi Province (20132BCD40026).

\section{References}

1. Mirabello L, Troisi RJ and Savage SA: International osteosarcoma incidence patterns in children and adolescents, middle ages and elderly persons. Int J Cancer 125: 229-234, 2009.

2. Longhi A, Errani C, De Paolis M, Mercuri M and Bacci G: Primary bone osteosarcoma in the pediatric age: State of the art. Cancer Treat Rev 32: 423-436, 2006.

3. Chou AJ and Gorlick R: Chemotherapy resistance in osteosarcoma: Current challenges and future directions. Expert Rev Anticancer Ther 6: 1075-1085, 2006.

4. Schwartz CL, Gorlick R, Teot L, Krailo M, Chen Z, Goorin A, Grier HE, Bernstein ML and Meyers P; Children's Oncology Group: Multiple drug resistance in osteogenic sarcoma: INT0133 from the Children's Oncology Group. J Clin Oncol 25: 2057-2062, 2007.

5. Wafa $\mathrm{H}$ and Grimer RJ: Surgical options and outcomes in bone sarcoma. Expert Rev Anticancer Ther 6: 239-248, 2006.

6. Oertel S, Blattmann C, Rieken S, Jensen A, Combs SE, Huber PE, Bischof M, Kulozik A, Debus J and Schulz-Ertner D: Radiotherapy in the treatment of primary osteosarcoma - a single center experience. Tumori 96: 582-588, 2010.

7. Mirabello L, Troisi RJ and Savage SA: Osteosarcoma incidence and survival rates from 1973 to 2004: Data from the Surveillance, Epidemiology, and End Results Program. Cancer 115: 1531-1543, 2009.

8. Picci P, Mercuri M, Ferrari S, Alberghini M, Briccoli A, Ferrari C, Pignotti E and Bacci G: Survival in high-grade osteosarcoma: Improvement over 21 years at a single institution. Ann Oncol 21: 1366-1373, 2010
9. Arai K, Sakamoto R, Kubota D and Kondo T: Proteomic approach toward molecular backgrounds of drug resistance of osteosarcoma cells in spheroid culture system. Proteomics 13: 2351-2360, 2013.

10. Robert RS, Ottaviani G, Huh WW, Palla S and Jaffe N: Psychosocial and functional outcomes in long-term survivors of osteosarcoma: A comparison of limb-salvage surgery and amputation. Pediatr Blood Cancer 54: 990-999, 2010.

11. Shangguan WJ, Li H and Zhang YH: Induction of G2/M phase cell cycle arrest and apoptosis by ginsenoside $\mathrm{Rf}$ in human osteosarcoma MG 63 cells through the mitochondrial pathway. Oncol Rep 31: 305-313, 2014

12. Li Z, Yu Y, Sun S, Qi B, Wang W and Yu A: Niclosamide inhibits the proliferation of human osteosarcoma cell lines by inducing apoptosis and cell cycle arrest. Oncol Rep 33: 1763-1768, 2015.

13. Wei J, Zhu Y, Xu G, Yang F, Guan Z, Wang M and Fang Y: Oxymatrine extracted from Sophora flavescens inhibited cell growth and induced apoptosis in human osteosarcoma MG-63 cells in vitro. Cell Biochem Biophys 70: 1439-1444, 2014.

14. Ahmed S, Anuntiyo J, Malemud CJ and Haqqi TM: Biological basis for the use of botanicals in osteoarthritis and rheumatoid arthritis: A review. Evid Based Complement Alternat Med 2: 301-308, 2005

15. Li PP, He W, Yuan PF, Song SS, Lu JT and Wei W: Celastrol induces mitochondria-mediated apoptosis in hepatocellular carcinoma Bel-7402 cells. Am J Chin Med 43: 137-148, 2015.

16. Mi C, Shi H, Ma J, Han LZ, Lee JJ and Jin X: Celastrol induces the apoptosis of breast cancer cells and inhibits their invasion via downregulation of MMP-9. Oncol Rep 32: 2527-2532, 2014.

17. Ni H, Zhao W, Kong X, Li H and Ouyang J: NF-kappa B modulation is involved in celastrol induced human multiple myeloma cell apoptosis. PLoS One 9: e95846, 2014.

18. Zhao X, Gao S, Ren H, Huang H, Ji W and Hao J: Inhibition of autophagy strengthens celastrol-induced apoptosis in human pancreatic cancer in vitro and in vivo models. Curr Mol Med 14: 555-563, 2014.

19. Lee HW, Jang KS, Choi HJ, Jo A, Cheong JH and Chun KH: Celastrol inhibits gastric cancer growth by induction of apoptosis and autophagy. BMB Rep 47: 697-702, 2014.

20. Zhang J, Song J, Wu D, Wang J and Dong W: Hesperetin induces the apoptosis of hepatocellular carcinoma cells via mitochondrial pathway mediated by the increased intracellular reactive oxygen species, ATP and calcium. Med Oncol 32: 101, 2015.

21. Pieme C, Santosh G, Tekwu E, Askun T, Aydeniz H, Ngogang J, Bhushan S and Saxena A: Fruits and barks extracts of Zanthozyllum heitzii a spice from Cameroon induce mitochondrial dependent apoptosis and G0/G1 phase arrest in human leukemia HL-60 cells. Biol Res 47: 54, 2014.

22. Zhang K, Wang X, Wang C, Zheng H, Li T, Xiao S, Wang M, Fei C, Zhang L and Xue F: Investigation of quinocetone-induced mitochondrial damage and apoptosis in HepG2 cells and compared with its metabolites. Environ Toxicol Pharmacol 39: 555-567, 2015.

23. Green DR and Reed JC: Mitochondria and apoptosis. Science 281: 1309-1312, 1998

24. Landriscina M, Laudiero G, Maddalena F, Amoroso MR, Piscazzi A, Cozzolino F, Monti M, Garbi C, Fersini A, Pucci P, et al: Mitochondrial chaperone Trap1 and the calcium binding protein Sorcin interact and protect cells against apoptosis induced by antiblastic agents. Cancer Res 70: 6577-6586, 2010.

25. Gross A, McDonnell JM and Korsmeyer SJ: BCL-2 family members and the mitochondria in apoptosis. Genes Dev 13: 1899-1911, 1999.

26. Manfredi G, Kwong JQ, Oca-Cossio JA, Woischnik M, Gajewski CD, Martushova K, D'Aurelio M, Friedlich AL and Moraes CT: BCL-2 improves oxidative phosphorylation and modulates adenine nucleotide translocation in mitochondria of cells harboring mutant mtDNA. J Biol Chem 278: 5639-5645, 2003.

27. Chang HY and Yang X: Proteases for cell suicide: Functions and regulation of caspases. Microbiol Mol Biol Rev 64: 821-846, 2000.

28. Cao X, Bennett RL and May WS: c-Myc and caspase-2 are involved in activating Bax during cytotoxic drug-induced apoptosis. J Biol Chem 283: 14490-14496, 2008.

29. Stennicke HR and Salvesen GS: Properties of the caspases. Biochim Biophys Acta 1387: 17-31, 1998.

30. Hui KK, Kanungo AK, Elia AJ and Henderson JT: Caspase-3 deficiency reveals a physiologic role for Smac/DIABLO in regulating programmed cell death. Cell Death Differ 18: 1780-1790, 2011. 
31. Volkmann N, Marassi FM, Newmeyer DD and Hanein D: The rheostat in the membrane: BCL-2 family proteins and apoptosis. Cell Death Differ 21: 206-215, 2014

32. Renault TT and Chipuk JE: Death upon a kiss: Mitochondrial outer membrane composition and organelle communication govern sensitivity to BAK/BAX-dependent apoptosis. Chem Biol 21: 114-123, 2014.

33. Westphal D, Kluck RM and Dewson G: Building blocks of the apoptotic pore: How Bax and Bak are activated and oligomerize during apoptosis. Cell Death Differ 21: 196-205, 2014.

34. Chen PM, Cheng YW, Wu TC, Chen CY and Lee H: MnSOD overexpression confers cisplatin resistance in lung adenocarcinoma via the NF- $\kappa \mathrm{B} / \mathrm{Snail} / \mathrm{Bcl}-2$ pathway. Free Radic Biol Med 79: 127-137, 2015.

35. Donskow-Łysoniewska K, Brodaczewska K and Doligalska M: Heligmosomoides polygyrus antigens inhibit the intrinsic pathway of apoptosis by overexpression of survivin and Bcl-2 protein in CD4 T cells. Prion 7: 319-327, 2013.

36. Hengartner MO: The biochemistry of apoptosis. Nature 407: 770-776, 2000.
37. Gillies LA and Kuwana T: Apoptosis regulation at the mitochondrial outer membrane. J Cell Biochem 115: 632-640, 2014.

38. Jourdain A and Martinou JC: Mitochondrial outer-membrane permeabilization and remodelling in apoptosis. Int $\mathrm{J}$ Biochem Cell Biol 41: 1884-1889, 2009.

39. Wood WG, Igbavboa U, Muller WE and Eckert GP: Statins, Bcl-2, and apoptosis: Cell death or cell protection? Mol Neurobiol 48: 308-314, 2013

40. Fulda $\mathrm{S}$ and Debatin KM: Extrinsic versus intrinsic apoptosis pathways in anticancer chemotherapy. Oncogene 25: 4798-4811, 2006.

41. Villa-Morales M and Fernández-Piqueras J: Targeting the Fas/FasL signaling pathway in cancer therapy. Expert Opin Ther Targets 16: 85-101, 2012.

42. Gordon $\mathrm{N}$ and Kleinerman ES: Aerosol therapy for the treatment of osteosarcoma lung metastases: Targeting the Fas/FasL pathway and rationale for the use of gemcitabine. J Aerosol Med Pulm Drug Deliv 23: 189-196, 2010. 